\title{
ESTADO DA ARTE SOBRE ENTEROPARASITOS EM COMUNIDADES INDÍGENAS DO BRASIL
}

\author{
THAYSE MACEDO DOS SANTOS LIMA ${ }^{1}$
}

IF Baiano

MARCELLA GOMEZ PEREIRA ${ }^{2}$

$U E S B$

ARTUR GOMES DIAS LIMA ${ }^{3}$

$E B M S P / U N E B$

\begin{abstract}
RESUMO: O objetivo dessa pesquisa foi realizar uma revisão sistemática da literatura acerca das pesquisas científicas sobre a ocorrência de enteroparasitoses em povos indígenas no Brasil, de acordo com as diretrizes PRISMA. Para a pesquisa, foram usadas as bases de dados Scielo, LILACS, MEDLINE via PubMed, Embase, Scopus, Bireme, Biblioteca de Manguinhos, Biblioteca Lincoln de Freitas Filho e o banco de teses da Universidade de São Paulo (USP). Foram selecionadas 57 produções científicas; dessas, 46 artigos, oito dissertações, uma tese, uma monografia e um relatório técnico. Os estudos sobre enteroparasitoses em indígenas aumentaram significativamente nos últimos anos; entretanto, a maioria foi desenvolvida na região Norte $e$ Centro-Oeste. Isso demonstra uma escassez de pesquisas sobre a temática nas demais regiões do país, a exemplo do Nordeste, que possui um grande número de etnias distribuídas em seu território e em que, ainda assim, poucos trabalhos vêm sendo desenvolvidos.
\end{abstract}

PALAVRAS-CHAVE: Populações indígenas; enteroparasitismo; produção científica.

ABSTRACT: This research aims to carry out a systematic review of literature on scientific researches over the occurrence of intestinal parasitic infections among indigenous people in Brazil, according to the PRISMA guidelines. For the research, databases were used, such as Scielo, LILACS, MEDLINE via PubMed, Embase, Scopus, Bireme, Manguinhos Library, Lincoln Freitas Filho Library and the thesis bank of the University of São Paulo (USP). We selected 57 scientific studies, of which 46 were articles, 8 were dissertations, one was a doctoral thesis, one

\footnotetext{
${ }^{1}$ Graduação em Ciência Biológicas pela Universidade do Estado da Bahia, Especialista em Saúde Pública pela Faculdade Nobre e Mestre em Ecologia Humana e Gestão Socioambiental pela Universidade do Estado da Bahia (2013-2015). Técnica Educacional do Instituto Federal Baiano. E-mail: thaymacedo@hotmail.com.

${ }^{2}$ Graduação em Ciência Biológicas pela Universidade Estadual de Santa Cruz, Mestre em Ecologia Humana e Gestão Socioambiental pela Universidade do Estado da Bahia (2013-2015). Atualmente é doutoranda do programa em Memória: Linguagem e Sociedade da Universidade do Estado da Bahia (UESB), da qual é bolsista CAPES. E-mail: pereira.gomez@ hotmail.com .

${ }^{3}$ Mestre em Entomologia pelo Instituto Nacional de Pesquisas da Amazônia/INPA-AM e Doutor em Biologia Parasitária pelo Instituto Oswaldo Cruz/IOC/FIOCRUZ. Professor Adjunto da Escola Bahiana de Medicina e Saúde Pública/EBMSP e Professor Pleno do Departamento de Ciências da Vida da Universidade do Estado da Bahia/DCV/UNEB. E-mail: parasitologista@gmail.com .
} 
was a graduation monography and one was a technical report. Studies on intestinal parasitic infections among indigenous people have increased significantly in recent years; however, most have been developed in the North and Midwest region. This demonstrates a lack of research on the subject in other regions of the country, like the Northeast, which possess a large number of ethnic groups distributed in its territory, but few studies developed.

KEYWORDS: Indigenous people; intestinal parasites; scientific production.

\section{Introdução}

Atualmente, a organização da estrutura básica para atendimento à saúde de povos indígenas no Brasil reflete os infortúnios da trajetória histórica desses sujeitos, a qual é marcada por conflitos fundiários associados à expansão das fronteiras demográficas nacionais, à degradação ambiental de áreas de preservação cultural e à omissão do Estado frente aos direitos dos indígenas como cidadãos brasileiros (COIMBRA, 2014). Estudos realizados com diferentes etnias a partir dos anos de 1990 destacaram o contexto das desigualdades sociais em saúde, que marcam a fronteira entre ser indígena e não indígena no Brasil (COIMBRA e SANTOS, 2000; SANTOS e COIMBRA, 2008). Os dados disponíveis, embora precários, apresentam taxas de morbidade e mortalidade de três a quatro vezes maiores que as encontradas na população brasileira em geral (COIMBRA, SANTOS e ESCOBAR, 2005).

A atenção básica à saúde indígena é uma área relevante para as pesquisas em enteroparasitoses, sobretudo para o desenvolvimento e a implementação de políticas públicas voltadas à qualidade de vida e da saúde indígena. Sendo o conhecimento das condições da saúde de populações indígenas fundamental para ações de prevenção e controle de doenças negligenciadas e endêmicas, as contribuições teóricoepistemólogicas sobre o tema representam uma valiosa contribuição aos programas de saúde implantados pelo Ministério da Saúde, sob responsabilidade da FUNASA (Fundação Nacional de Saúde), através da Coordenação do Índio (COSAI) e dos Distritos Sanitários Especiais Indígenas (DSEI) (SOUZA, SCATENA e SANTOS, 2007). 
De acordo com Leite et al (2007), no Brasil, ser indígena implica maiores chances de óbito durante o primeiro ano de vida, de desnutrição e anemia durante o período de crescimento, de convivência com elevada carga de doenças infecciosas e parasitárias e de exposição ao rápido processo de transição nutricional, o qual é responsável pela emergência de agravos como obesidade, hipertensão arterial e diabetes mellitus (COIMBRA e BASTA, 2007; CARDOSO, Coimbra e WERNEK, 2013). Entre as doenças infecto-parasitárias no perfil de morbimortalidade indígena estão as endemias (tuberculose, malária e leishmaniose), gastroenterites e infecções respiratórias (COIMBRA e SANTOS, 2001). A prevalência e o espectro do enteroparasitismo apresentam variações de acordo com a localidade, em decorrência de fatores climáticos, socioeconômicos, educacionais e de saneamento (KOBAYASHI et al, 1995).

As enteroparasitoses podem interferir na absorção de nutrientes e causar complicações significativas, como obstrução intestinal, prolapso retal e formação de abcessos. As enteroparasitoses apresentam padrões de morbidade significativos, já que a presença de múltiplos parasitas, associados a estados de carência e desnutrição graves, pode causar o óbito do indivíduo infectado (REY, 2002).

A revisão sistemática sobre a produção científica acerca da ocorrência de enteroparasitoses em indígenas brasileiros permite apresentar as dimensões geográficas, econômicas e sociais das questões que envolvem a saúde indígena no Brasil. Nesse contexto, o referido trabalho objetivou, a partir de uma revisão bibliográfica sistemática da literatura em saúde pública acerca das pesquisas científicas sobre o enteroparasitismo em populações indígenas no Brasil, organizar os dados disponíveis sobre a temática a fim de contribuir para os órgãos responsáveis pela saúde indígena no Brasil em suas futuras ações quanto ao desenvolvimento, à implementação e à gestão de políticas públicas voltadas a saúde indígena.

\section{Material e métodos}

Este trabalho de pesquisa foi realizado através da revisão bibliográfico-documental, em repositórios virtuais, dos estudos sobre a 
ocorrência de enteroparasitoses em indígenas brasileiros. A revisão foi conduzida de acordo com as orientações do Preferred Reporting Items for Systematic Reviews and Meta Analyses (PRISMA) (MOHER et al, 2009) (Figura 01).

Foram analisadas as produções científicas publicadas no período de 1952 a 2016, sob a forma de artigos científicos, teses, dissertações, monografias e relatórios. Os artigos publicados em periódicos indexados foram localizados através das seguintes bases de dados: SciELO, LILACS, MEDLINE via PubMed, Embase, Scopus e Bireme. A pesquisa de teses e dissertações foi realizada através do sistema de busca da Biblioteca de Manguinhos, Biblioteca Lincoln de Freitas Filho, ambas da Fundação Oswaldo Cruz (Fiocruz), e do banco de teses da USP, que possuem catálogos de teses e dissertações de várias instituições.

Figura 01: Fluxograma da produção cientifica selecionada na revisão sistemática

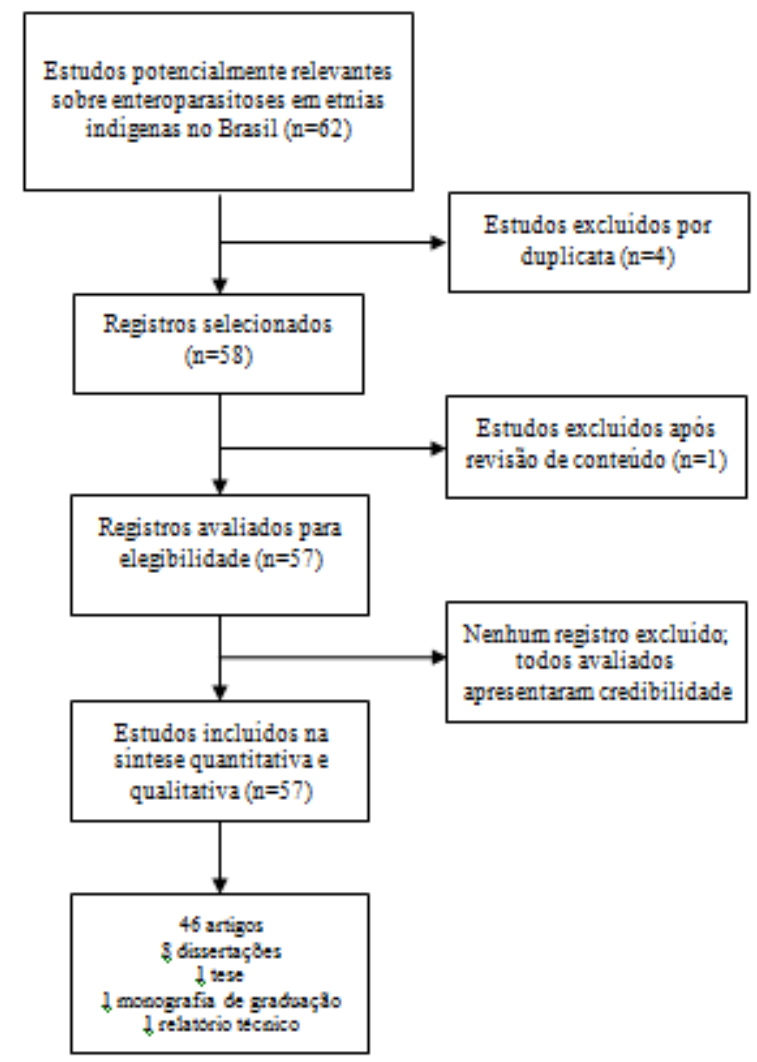

Fonte: Adaptada de Prisma group (MOHER et al, 2009), por Thayse Macedo. 
As palavras-chave utilizadas foram: doenças parasitárias, enteroparasitoses, parasitoses intestinais, índios sul-americanos, epidemiologia, prevalência, incidência, parasitologia, saneamento, higiene, Brasil, América Latina e populações indígenas. E, também, suas respectivas traduções na língua inglesa: parasitic diseases, intestinal parasites, South-american indians, epidemiology, prevalence, incidence, parasitology, sanitation, hygiene, Brazil, Latin America, native Southamerican indians.

\section{Resultados}

Foram encontradas e analisadas 57 produções científicas (Tabela 01) referentes à pesquisa sobre enteroparasitismo em populações indígenas no Brasil entre os anos 1952 e 2016 . Do total, 46 foram artigos publicados em periódicos, oito dissertações, uma tese, uma monografia de graduação e um relatório técnico.

A publicação mais antiga é a de Oliveira (1952), O estado de saúde dos índios Karajás em 1950. Nessa pesquisa, o autor tratou da saúde indígena no geral, incluindo uma análise de exames parasitológicos. $\mathrm{Na}$ década de 1960 e 1970, foram escassas as publicações, com duas e quatro respectivamente, havendo um aumento significativo nas décadas seguintes: na década de 1980, foram sete publicações; nos anos 1990, foram dez publicações registradas e, nos anos de 2000, houve trinta e três publicações sobre a ocorrência de enteroparasitoses em indígenas brasileiros.

Sobre a abrangência geográfica das pesquisas (Figura 02) de enteroparasitoes em indígenas brasileiros, o presente estudo revelou que foram desenvolvidas pesquisas com essa temática nas cinco regiões do país, destacando-se a região Norte, com 49\% das produções, seguida pelas regiões Centro-Oeste, com 23\%, Sul e Sudeste, com 13\% cada. 0 Nordeste apresentou apenas $3 \%$ do total das bibliografias encontradas. 
Santos Lima, Pereira e Dias Lima - Estado da arte sobre enteroparasitos em comunidades...

Figura 02: Abrangência geográfica das produções científicas sobre a presença de enteroparasitos em comunidades indígenas nas diferentes regiões do Brasil

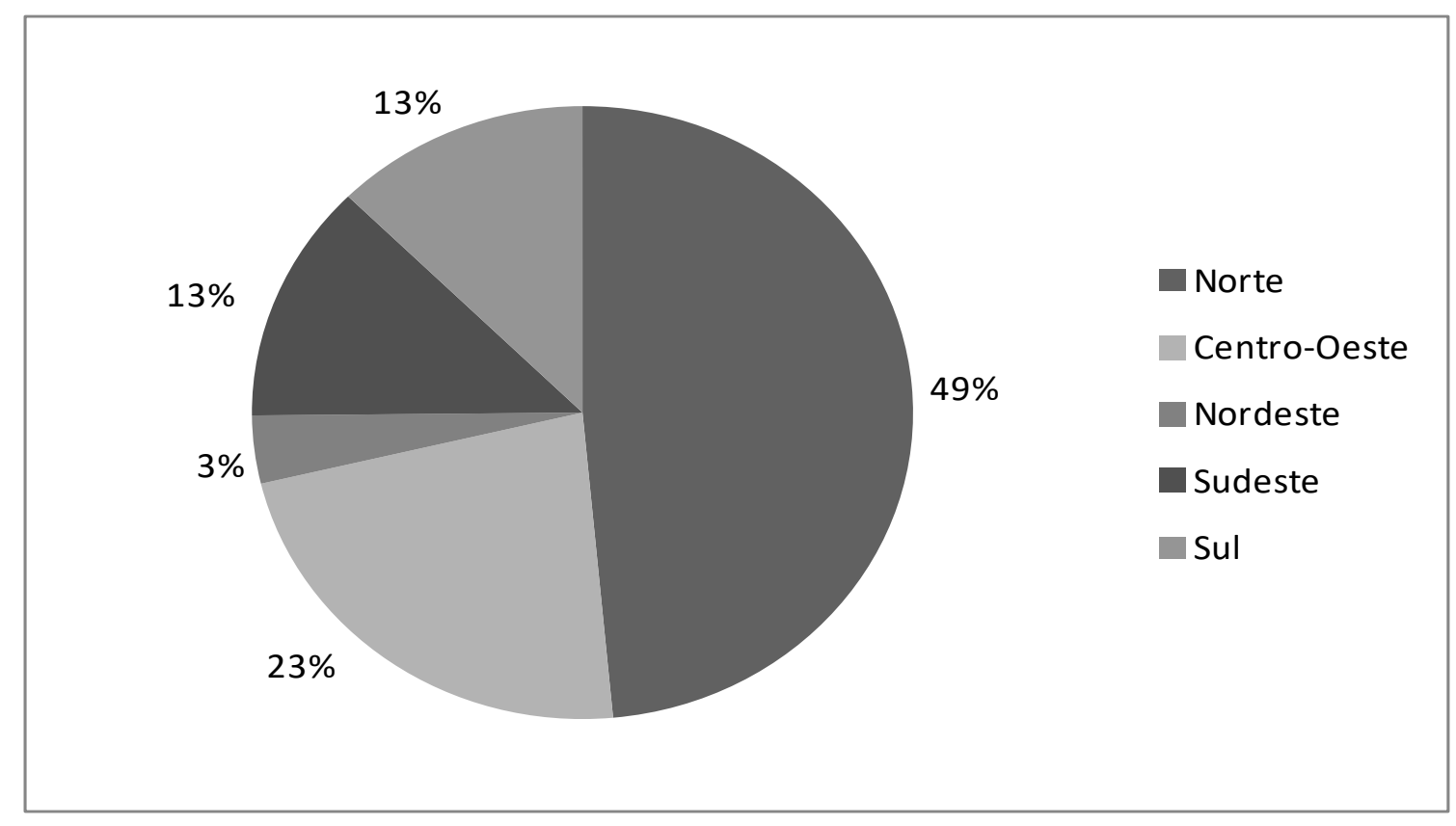

Fonte: autoria de Thayse Macedo a partir dos dados do próprio artigo.

Tabela 1: Produções científicas que abordam a ocorrência de enteroparasitoses em indígenas no Brasil

\begin{tabular}{|c|c|c|c|c|c|c|}
\hline Autor/Ano & $\begin{array}{c}\text { Tipo de } \\
\text { produção }\end{array}$ & Estado & Etnia & Técnica & Protozoários & Helmintos \\
\hline $\begin{array}{c}\text { Oliveira, H. } \\
(1952)\end{array}$ & Artigo & Tocantins & Karajá & NI & $\mathrm{NI}$ & $A l$ \\
\hline $\begin{array}{c}\text { Aytai, D. } \\
\text { (1966) }\end{array}$ & Artigo & $\begin{array}{c}\text { Mato } \\
\text { Grosso }\end{array}$ & Mamaindê & NI & NI & $A l, A n c$ \\
\hline $\begin{array}{c}\text { Neel et al } \\
(1968)\end{array}$ & Artigo & $\begin{array}{c}\text { Mato } \\
\text { Grosso }\end{array}$ & Xavante & $\begin{array}{c}\text { Ritchie } \\
\text { (centrifugação } \\
\text { por formalina- } \\
\text { éter) }\end{array}$ & $\begin{array}{l}G l, C m, E c, \\
E h, E m, I b\end{array}$ & $\begin{array}{c}A l, A n c, S s, \\
\quad T t, E v\end{array}$ \\
\hline $\begin{array}{c}\text { Knight \& } \\
\text { Prata (1972) }\end{array}$ & Artigo & Amazonas & NE & $\begin{array}{c}\text { Exame direto, } \\
\text { Ritchie } \\
\text { (concentração } \\
\text { por formol-éter) }\end{array}$ & $\begin{array}{c}E n h, G l, E c, \\
I b, C m, E n, \\
E h\end{array}$ & $\begin{array}{c}A n c, A l, T t, \\
S s\end{array}$ \\
\hline $\begin{array}{l}\text { Perez et al } \\
\text { (1972) }\end{array}$ & Artigo & São Paulo & $\begin{array}{c}\text { Guarani, } \\
\text { Kaiangang e } \\
\text { Terena }\end{array}$ & $\begin{array}{l}\text { Lutz (Hoffman), } \\
\text { Faust e Willis }\end{array}$ & $\begin{array}{c}G l, E h, E c, \\
E s p ., \quad I b, E n, \\
C m, E n\end{array}$ & $\begin{array}{c}T s p ., H n, A l, \\
A n c, S s, E v, \\
T t, S m\end{array}$ \\
\hline $\begin{array}{c}\text { Fagundes } \\
\text { Neto (1977) }\end{array}$ & Artigo & $\begin{array}{l}\text { Mato } \\
\text { Grosso }\end{array}$ & $\begin{array}{c}\text { Aweti, } \\
\text { Waurá, } \\
\text { Kalapalo, } \\
\text { Kuikuro, } \\
\text { Yawalapiti, }\end{array}$ & $\begin{array}{c}\text { Harada-Mori e } \\
\text { Kato-Katz }\end{array}$ & $\begin{array}{c}E c, \quad G 1, I b, \\
\quad E h, C m\end{array}$ & $\begin{array}{l}\text { Anc, } A l, \\
S s, \quad T t, \quad E v\end{array}$ \\
\hline
\end{tabular}


Santos Lima, Pereira e Dias Lima - Estado da arte sobre enteroparasitos em comunidades...

\begin{tabular}{|c|c|c|c|c|c|c|}
\hline & & & $\begin{array}{l}\text { Mehinako, } \\
\text { Matipu- } \\
\text { Nahukwá, } \\
\text { Trumai } \\
\end{array}$ & & & \\
\hline $\begin{array}{l}\text { Bruno } \\
(1978)\end{array}$ & Dissertação & Amapá & $\begin{array}{l}\text { Karipuna, } \\
\text { Palikur, } \\
\text { Galibi }\end{array}$ & Exame Direto & $\begin{array}{c}E h, E c, E n, \\
I b, G l, E t h, E c\end{array}$ & $\begin{array}{c}A l, H n, \\
A n c, T t, S s, \\
E v\end{array}$ \\
\hline $\begin{array}{l}\text { Lawrence et } \\
\text { al (1980) }\end{array}$ & Artigo & Amazonas & $\begin{array}{c}\text { Yanomami e } \\
\text { Kaxinawá }\end{array}$ & $\begin{array}{c}\text { Exame Direto e } \\
\text { Ritchie } \\
\text { (concentração } \\
\text { por formalina- } \\
\text { éter }\end{array}$ & $\begin{array}{l}E c, E h, E t h, \\
I b, E n h, B c \\
G l, C m, D f\end{array}$ & $\begin{array}{c}A l, A n c, S s, \\
T t\end{array}$ \\
\hline $\begin{array}{l}\text { Coimbra Jr. } \\
\text { et.al (1981) }\end{array}$ & Artigo & Rondônia & Suruí & $\begin{array}{l}\text { Lutz (Hoffman), } \\
\text { Faust e Willis }\end{array}$ & $G l, E h$ & $\begin{array}{c}A l, A n c, S s, \\
T s p ., T t, H n, \\
E v\end{array}$ \\
\hline $\begin{array}{c}\text { Genaro \& } \\
\text { Ferraroni } \\
(1984)\end{array}$ & Artigo & Amazonas & Nadeb-Maku & Ritchie & $\begin{array}{l}E c, E m, G l \\
E h, C m, P h\end{array}$ & $\begin{array}{c}A l, A n c, T t, \\
S s\end{array}$ \\
\hline $\begin{array}{c}\text { Santos et al } \\
(1985)\end{array}$ & Artigo & Rondônia & $\begin{array}{c}\text { Pakaanovo, } \\
\text { Massaká, } \\
\text { Ajuru, } \\
\text { Canoe, } \\
\text { Jaboti, } \\
\text { Mequém, } \\
\text { Tupari e } \\
\text { Macurap } \\
\end{array}$ & $\begin{array}{c}\text { Lutz } \\
\text { (sedimentação) }\end{array}$ & $\begin{array}{l}G l, E h, E c, \\
C m, I b, E n\end{array}$ & $\begin{array}{c}A l, A n c, \\
S s, E v, \\
T t, ., H n\end{array}$ \\
\hline $\begin{array}{l}\text { Kameyama } \\
\text { (1985) }\end{array}$ & Dissertação & $\begin{array}{l}\text { Mato } \\
\text { Grosso }\end{array}$ & $\begin{array}{c}\text { Aweti, } \\
\text { Kalapalo, } \\
\text { Kamayurá, } \\
\text { Kuikuru, } \\
\text { Yawalapiti, } \\
\text { Mehinaku, } \\
\text { Nahukwá, } \\
\text { Matipu, } \\
\text { Waurá, } \\
\text { Kayabi, } \\
\text { Juruna, Suyá, } \\
\text { Trumai }\end{array}$ & $\begin{array}{l}\text { Lutz (Hoffman), } \\
\text { Faust, Willis, } \\
\text { Stool \& } \\
\text { Hausseer }\end{array}$ & $\begin{array}{c}G l, C m, E h, \\
\quad E n h, E c\end{array}$ & $\begin{array}{c}A l, S s, T t \\
A n c, E v\end{array}$ \\
\hline $\begin{array}{l}\text { Confalonieri } \\
\text { et a (1989) }\end{array}$ & Artigo & Roraima & Yanomami & Lutz & $E h, G l$ & $\begin{array}{l}A l, A n c, T t, \\
\quad S s, E v\end{array}$ \\
\hline $\begin{array}{c}\text { Chenela \& } \\
\text { Thatcher } \\
\text { (1989) }\end{array}$ & Artigo & Amazonas & $\begin{array}{l}\text { Tukano e } \\
\text { Maku }\end{array}$ & $\begin{array}{c}\text { Faust } \\
\text { (microflutuação), } \\
\text { Harada-Mori e } \\
\text { Lutz }\end{array}$ & $\begin{array}{c}E c, C m, E h \\
E n, I b, E t h, \\
G l, B c\end{array}$ & $\begin{array}{l}T t, A l, N a \\
\quad E v, S s\end{array}$ \\
\hline $\begin{array}{c}\text { Coimbra Jr. } \\
\text { et al } \\
(1991-a) \\
\end{array}$ & Artigo & Rondônia & Suruí & $\begin{array}{c}\text { Lutz } \\
\text { (sedimentação) }\end{array}$ & $\begin{array}{l}G l, E h, E c \\
I b, C m, E m\end{array}$ & $\begin{array}{l}A n c, \quad A l, \\
S s, \quad T t, \quad E v \\
H n\end{array}$ \\
\hline $\begin{array}{l}\text { Ferreira et } \\
\text { al (1991) }\end{array}$ & Artigo & $\begin{array}{l}\text { Mato } \\
\text { Grosso }\end{array}$ & Yawalapiti & $\begin{array}{c}\text { Lutz (Hoffman), } \\
\text { Faust, Willis ou } \\
\text { Exame Dierto }\end{array}$ & $\begin{array}{c}E c, G l, E n, \\
I b\end{array}$ & $\begin{array}{c}A n c, A l, E v, \\
H n\end{array}$ \\
\hline $\begin{array}{l}\text { Coimbra Jr. } \\
\text { et al } \\
(1991-b)\end{array}$ & Artigo & $\begin{array}{l}\text { Mato } \\
\text { Grosso }\end{array}$ & Zoró & $\begin{array}{c}\text { Lutz } \\
\text { (sedimentação) }\end{array}$ & $G l, B c, E h$ & $A n c, T t, H n$ \\
\hline $\begin{array}{c}\text { Ferrari et al } \\
(1992)\end{array}$ & Artigo & Rondônia & Karitiana & $\begin{array}{l}\text { Lutz (Hoffman), } \\
\text { Faust, Willis }\end{array}$ & $E h, E c$ & $\begin{array}{c}A l, A n c, T t, \\
E v, H n\end{array}$ \\
\hline $\begin{array}{c}\text { Linhares } \\
(1992)\end{array}$ & Artigo & Amazonas & $\mathrm{NE}$ & NI & NI & NI \\
\hline $\begin{array}{l}\text { Santos et al } \\
\text { (1995) }\end{array}$ & Artigo & $\begin{array}{l}\text { Mato } \\
\text { Grosso }\end{array}$ & Xavante & $\begin{array}{c}\text { Lutz } \\
\text { (sediemntação) e } \\
\text { Simões Barbosa }\end{array}$ & $\begin{array}{c}E c, E h, E m \\
G l, I b .\end{array}$ & $\begin{array}{c}A l, A n c, S s, \\
T t, E v, H n\end{array}$ \\
\hline $\begin{array}{c}\text { Lanelli et al } \\
\text { (1995) }\end{array}$ & Artigo & $\begin{array}{l}\text { Mato } \\
\text { Grosso }\end{array}$ & Xavante & $\begin{array}{c}\text { Lutz } \\
\text { (sedimentação) }\end{array}$ & $\begin{array}{c}G l, E h, E c, \\
E m, I b\end{array}$ & $A l, A n c, S s$ \\
\hline
\end{tabular}


Santos Lima, Pereira e Dias Lima - Estado da arte sobre enteroparasitos em comunidades...

\begin{tabular}{|c|c|c|c|c|c|c|}
\hline $\begin{array}{l}\text { Serafim } \\
(1997)\end{array}$ & Artigo & $\begin{array}{c}\text { Amazonas, } \\
\text { Rondônia, } \\
\text { Pará }\end{array}$ & $\begin{array}{l}\text { Suruí, } \\
\text { Pacaánova, } \\
\text { Guaporé, } \\
\text { Mamoré, } \\
\text { Yanomámi, } \\
\text { Tiriyó }\end{array}$ & $\begin{array}{l}\text { Lutz (Hoffman), } \\
\text { Faust, Willis }\end{array}$ & $\begin{array}{l}G l, I b, E c \\
\quad E n, E h\end{array}$ & $\begin{array}{l}A l, T t, A n c, \\
\quad S s, H n\end{array}$ \\
\hline $\begin{array}{l}\text { Miranda et } \\
\text { al (1998) }\end{array}$ & Artigo & Pará & Parakanã & $\begin{array}{l}\text { Lutz (Hoffman), } \\
\text { Faust, Willis, } \\
\text { Exame Direto }\end{array}$ & $E h, G l$ & $\begin{array}{l}A l, A n c, T t, \\
\quad S s, E v\end{array}$ \\
\hline $\begin{array}{l}\text { Miranda et } \\
\text { al(1999) }\end{array}$ & Artigo & Pará & Tembé & $\begin{array}{l}\text { Lutz (Hoffman), } \\
\text { Faust, Willis, } \\
\text { Exame Direto }\end{array}$ & $G l, E h$ & $A l, A n c, T t$, \\
\hline $\begin{array}{l}\text { Scolari et al } \\
(2000)\end{array}$ & Artigo & Paraná & $\mathrm{NE}$ & Kato-Katz & SR & $A l, A n c, T t$ \\
\hline $\begin{array}{l}\text { Fontbone et } \\
\text { al (2001) }\end{array}$ & Artigo & Pernambuco & Pankararu & $\begin{array}{c}\text { Lutz } \\
\text { (sedimentação) }\end{array}$ & $E c, G l, E h, I b$ & $\begin{array}{c}A l, A n c, T t, \\
T s, S s\end{array}$ \\
\hline $\begin{array}{l}\text { Carme et a } \\
\text { (2002) }\end{array}$ & Artigo & Amapá & Waiãpi & $\begin{array}{c}\text { Exame Direto, } \\
\text { Kato-Katz e } \\
\text { Baermann \& } \\
\text { Moraes }\end{array}$ & $\begin{array}{l}G l, E h / E d, \\
\quad E c, C m\end{array}$ & $A l, T t, E v$ \\
\hline $\begin{array}{l}\text { Vieira } \\
(2003)\end{array}$ & Dissertação & $\begin{array}{l}\text { Rio de } \\
\text { Janeiro }\end{array}$ & NI & NI & SR & SR \\
\hline $\begin{array}{l}\text { Haverroth et } \\
\text { al (2003) }\end{array}$ & Relatório & Rondônia & $\mathrm{NE}$ & NI & SR & SR \\
\hline $\begin{array}{l}\text { Gilio et al } \\
\text { (2006) }\end{array}$ & Artigo & Paraná & $\begin{array}{l}\text { Kaiangang, } \\
\text { Rani e Xetá }\end{array}$ & $\begin{array}{l}\text { Lutz (Hoffman), } \\
\text { Ritchie }\end{array}$ & $G l, E h, E c, E n$ & $A l, T t, H n$ \\
\hline $\begin{array}{l}\text { Aguiar et al } \\
\text { (2007) }\end{array}$ & Artigo & $\begin{array}{c}\text { Mato } \\
\text { Grosso do } \\
\text { Sul } \\
\end{array}$ & Terena & $\begin{array}{c}\text { Lutz } \\
\text { (sedimentação } \\
\text { espontânea) } \\
\end{array}$ & $\begin{array}{l}G l, E h, E c, \\
E m, B h, I b\end{array}$ & $\begin{array}{c}A l, \mathrm{Na}, \mathrm{Ss} \\
\mathrm{Hn}\end{array}$ \\
\hline $\begin{array}{l}\text { Carvalho- } \\
\text { Costa et al } \\
(2007) \\
\end{array}$ & Artigo & Amazonas & $\begin{array}{c}\text { Tukano e } \\
\text { Aruak }\end{array}$ & $\begin{array}{l}\text { Ritchie, Kato- } \\
\text { Katz, Baermann- } \\
\text { Moraes }\end{array}$ & $G l, E h, B h$ & $\begin{array}{l}A l, \mathrm{Na}, \mathrm{Tt} \\
\quad \mathrm{Ss}, \mathrm{Hn}\end{array}$ \\
\hline $\begin{array}{l}\text { Rios et al } \\
(2007)\end{array}$ & Artigo & Amazonas & $\begin{array}{c}\text { Tariano, } \\
\text { Tukano, } \\
\text { Piratapuia, } \\
\text { Wanano, } \\
\text { Hupda, } \\
\text { Desana, Juriti }\end{array}$ & $\begin{array}{c}\text { Lutz } \\
\text { (sedimentação) }\end{array}$ & $\begin{array}{l}G l, H h, E c, \\
\quad E m, B h\end{array}$ & $\begin{array}{l}A l, \mathrm{Na}, \mathrm{Tt} \\
\mathrm{Ss}, \mathrm{Hn} \mathrm{Ev}\end{array}$ \\
\hline $\begin{array}{l}\text { Moreira } \\
(2008)\end{array}$ & Dissertação & $\begin{array}{l}\text { Minas } \\
\text { Gerais }\end{array}$ & Krenak & $\begin{array}{c}\text { TF-Teste }{ }^{\circledR} \\
\text { (centrifugação e } \\
\text { dupla fikltragem) }\end{array}$ & $G l, E h, E c$ & $\begin{array}{c}A l, A n c, S s, \\
H n\end{array}$ \\
\hline $\begin{array}{l}\text { Toledo et al } \\
\text { (2009) }\end{array}$ & Artigo & Paraná & Kaingang & $\begin{array}{c}\text { Lutz } \\
\text { (sedimentação } \\
\text { espontânea), } \\
\text { kato-katz }\end{array}$ & $\begin{array}{l}G l, E h, E c \\
\quad E m, I b\end{array}$ & $\begin{array}{c}A l, A n c, T t, \\
H n, S s, E v, \\
T s\end{array}$ \\
\hline $\begin{array}{l}\text { Borges et al } \\
\quad(2009)\end{array}$ & Artigo & Pará & Oriximiná & $\begin{array}{l}\text { Lutz, Exame } \\
\text { Direto, } \\
\text { Centrífugo- } \\
\text { flutuação } \\
\text { (sulfato de } \\
\text { zinco) } \\
\end{array}$ & $\begin{array}{l}G l, E c, E h, \\
\quad E n, I b\end{array}$ & $A l, E v, H n$ \\
\hline $\begin{array}{l}\text { Boia et al } \\
(2009)\end{array}$ & Artigo & Amazonas & $\begin{array}{c}\text { Tariana, } \\
\text { Baniwa e } \\
\text { Hupda } \\
\end{array}$ & Croprotest $®$ & $G l, E h$ & $A l, T t, A n c$ \\
\hline $\begin{array}{l}\text { Zardinello } \\
\text { et al (2009) }\end{array}$ & Artigo & Paraná & Avá Guarani & $\begin{array}{l}\text { Faust e Lutz } \\
\text { (Hoffman) }\end{array}$ & $\begin{array}{l}G l, E h, E c, \\
\quad E m, I b\end{array}$ & $A n c, H n, S s$ \\
\hline $\begin{array}{l}\text { Palhano- } \\
\text { Silva et al } \\
\text { (2009) }\end{array}$ & Artigo & Amazonas & Suruí & $\begin{array}{c}\text { Lutz } \\
\text { (sedimentação), } \\
\text { Flutuação } \\
\text { (sulfato de } \\
\text { zinco) }\end{array}$ & $\begin{array}{l}G l, E h, E c, \\
E m, I b, B h\end{array}$ & $A n c, H n, S s$ \\
\hline
\end{tabular}


Santos Lima, Pereira e Dias Lima - Estado da arte sobre enteroparasitos em comunidades...

\begin{tabular}{|c|c|c|c|c|c|c|}
\hline $\begin{array}{l}\text { Moura et al } \\
(2010)\end{array}$ & Artigo & Paraná & Kaingang & $\begin{array}{c}\text { Lutz } \\
\text { (sedimentação), } \\
\text { Baermann, Faust }\end{array}$ & $\begin{array}{c}G l, E s p, E m, \\
B h\end{array}$ & $\begin{array}{c}A l, T t, A n c, \\
T s, S s, H n,\end{array}$ \\
\hline $\begin{array}{l}\text { Pardo et al } \\
(2010)\end{array}$ & Artigo & Amazonas & NI & Lutz (Hoffman) & $G l, E c, E h, I b$ & $\begin{array}{c}A l, H n, S m, \\
A n c\end{array}$ \\
\hline Assis (2010) & Dissertação & $\begin{array}{l}\text { Minas } \\
\text { Gerais }\end{array}$ & Maxakali & $\begin{array}{c}\text { TF-Teste } \circledR \\
\text { (centrifugação e } \\
\text { dupla filtragem) } \\
\end{array}$ & $\begin{array}{c}G l, E c, E m, \\
E h\end{array}$ & $\begin{array}{c}A l, S s, A n c, \\
T t, H n, S m\end{array}$ \\
\hline Silva (2010) & Dissertação & $\begin{array}{l}\text { Mato } \\
\text { Grosso }\end{array}$ & Xavante & $\begin{array}{c}\text { Lutz } \\
\text { (sedimentação) } \\
\text { Kato-Katz } \\
\end{array}$ & $G l, E c, E h, E n$ & $\begin{array}{c}A l, A n c, H n, \\
E v, T t, E s\end{array}$ \\
\hline $\begin{array}{l}\text { Neves } \\
(2010)\end{array}$ & Dissertação & São Paulo & Guarani Mbya & $\begin{array}{l}\text { Lutz (Hoffman), } \\
\text { Exame direto }\end{array}$ & $G l, E c, E n$ & $A l, H n$ \\
\hline $\begin{array}{l}\text { Carvalho } \\
\text { (2011) }\end{array}$ & Dissertação & $\begin{array}{l}\text { Minas } \\
\text { Gerais }\end{array}$ & Xakriabá & $\begin{array}{c}\text { TF-Teste }{ }^{\circledR} \\
\text { (centrifugação e } \\
\text { sedimentação) }\end{array}$ & $\begin{array}{c}G l, E h, E c, I b, \\
E n\end{array}$ & $\begin{array}{c}A l, A n c, S s, \\
T s, H n, S m, \\
T t, E v\end{array}$ \\
\hline $\begin{array}{c}\text { Pardo et al } \\
(2011)\end{array}$ & Artigo & Amazonas & $\begin{array}{c}\text { Ikpeng, } \\
\text { Kaiabi, kisêjê }\end{array}$ & Lutz (Hoffmann) & $G l, E c, I b$ & $A l, A n c, H n$ \\
\hline $\begin{array}{l}\text { Malheiros } \\
\text { (2011) }\end{array}$ & Tese & $\begin{array}{l}\text { Mato } \\
\text { Grosso }\end{array}$ & Tapirapé & $\begin{array}{c}\text { Lutz (Hoffman), } \\
\text { Ritchie, } \\
\text { Coloração de } \\
\text { Kinyoun a frio } \\
\end{array}$ & $\begin{array}{l}G l, E c, E h, \\
\quad E m, B h\end{array}$ & $\begin{array}{c}A l, A n c, S s, \\
H n\end{array}$ \\
\hline $\begin{array}{l}\text { Scherette } \\
(2011)\end{array}$ & Monografia & $\begin{array}{c}\text { Rio grande } \\
\text { do Sul }\end{array}$ & NI & NI & SR & SR \\
\hline $\begin{array}{l}\text { Brandelli et } \\
\text { al (2012) }\end{array}$ & Artigo & $\begin{array}{l}\text { Rio Grande } \\
\text { do Sul }\end{array}$ & $\begin{array}{l}\text { Mbyá- } \\
\text { Guarani }\end{array}$ & $\begin{array}{c}\text { Lutz } \\
\text { (sedimentação } \\
\text { espontânea), } \\
\text { Centrifugo- } \\
\text { flutuação e Kato- } \\
\text { Katz } \\
\end{array}$ & $G l, E h, E c, E n$ & $\begin{array}{c}A l, E v, H n, \\
S s, A n c, T t\end{array}$ \\
\hline $\begin{array}{l}\text { Junior et al } \\
\text { (2013) }\end{array}$ & Artigo & $\begin{array}{l}\text { Minas } \\
\text { Gerais }\end{array}$ & Caxixó & $\begin{array}{c}\text { TF-Teste }{ }^{\circledR} \\
\text { Centrifugação- } \\
\text { flutuação }\end{array}$ & $G l, E h, E c$ & $A l, A n c, S s$ \\
\hline $\begin{array}{c}\text { Cerqueira et } \\
\text { al (2013) }\end{array}$ & Artigo & $\begin{array}{l}\text { Mato } \\
\text { Grosso }\end{array}$ & Xavante & NI & SR & SR \\
\hline $\begin{array}{l}\text { Oliveira et } \\
\text { al (2013) }\end{array}$ & Artigo & Bahia & Pankararé & $\begin{array}{l}\text { Lutz (Hoffman), } \\
\text { Willis, Kinyoun }\end{array}$ & $G l, E h, E c, E n$ & $A n c, H n$ \\
\hline $\begin{array}{l}\text { Andrade et } \\
\text { al (2013) }\end{array}$ & Artigo & Pará & Kayapós & Lutz (Hoffman) & $G l, E c, E n$ & $A l, H n$ \\
\hline $\begin{array}{l}\text { Malheiros et } \\
\text { al (2014) }\end{array}$ & Artigo & $\begin{array}{l}\text { Mato } \\
\text { Grosso }\end{array}$ & Tapirapé & $\begin{array}{c}\text { Microscopia } \\
\text { direta }\end{array}$ & SR & Ss \\
\hline $\begin{array}{l}\text { Malheiros et } \\
\text { al (2014) }\end{array}$ & Artigo & Amazonas & Tapirapé & $\begin{array}{c}\text { Lutz (Hoffmann) } \\
\text { e Ritchie }\end{array}$ & $H n$ & SR \\
\hline $\begin{array}{c}\text { Neres- } \\
\text { Norberg et } \\
\text { al (2014) }\end{array}$ & Artigo & $\begin{array}{c}\text { Mato } \\
\text { Grosso do } \\
\text { Sul } \\
\end{array}$ & Terena & $\begin{array}{c}\text { Lutz } \\
\text { (Hoffman); } \\
\text { Willis, Kinyoun }\end{array}$ & $\begin{array}{l}\mathrm{Cr}, \mathrm{Gl}, \mathrm{En}, \\
\text { Eh, Ec }\end{array}$ & $\begin{array}{c}\text { Al, Ev, Ss, } \\
\text { Tt, Anc, } \\
\text { Hn, Ts }\end{array}$ \\
\hline $\begin{array}{l}\text { Oliveira et } \\
\text { al (2016) }\end{array}$ & Artigo & Amazonas & $\begin{array}{l}\text { Baré e } \\
\text { Baniwa }\end{array}$ & $\begin{array}{c}\text { Lutz (Hoffmann) } \\
\text { e Ritchie }\end{array}$ & $\begin{array}{c}\text { Gl, Ec, Esp, } \\
\text { En, Ib, Bc }\end{array}$ & $\begin{array}{c}\text { Al, Anc, } \\
\mathrm{Hn}, \mathrm{Tt}, \mathrm{Ev}\end{array}$ \\
\hline
\end{tabular}

(NI) Não indicado

(NE) Não especificado

(SR) Sem resultado

Al: Ascaris lumbricoides; Anc: Ancilostomídeos; Bc: Balantidium coli; Bh: Blastocystis hominis; $\mathrm{Cm}$ : Chilomastix mesnili; Cr: Cryptosporidium spp; Ec: Entamoeba coli; En: Endolimax nana; Eh: Entamoeba histolytica; Enh: Entamoeba hartmanni; Esp: Entamoeba sp; Eth: Enteromonas hominis; Ev: Enterobius vermicularis; Gl: Giardia lamblia; Hn: Hymenolepis nana; Ib: Iodamoeba bütschlii; Na: Necator americanus; Ph: Pentatrichomonas hominis; Ss: Strongyloides stercoralis; Sm: Schistosoma mansoni; Ts: Taenia saginata; Tsp: Taenia sp. OBS: Lutz: As técnicas descritas pelos autores como Hoffmann, Pons e Janer, sedimentação de Lutz e as referidas como "Lutz". 
De acordo com a Tabela 01 , os trabalhos de pesquisa sobre a ocorrência de enteroparasitoses em indígenas brasileiros foram desenvolvidos em mais de uma comunidade ao mesmo tempo. A partir da sistematização do referencial bibliográfico documental, foi possível apresentar aqui os sujeitos de pesquisa mais requisitados. Entre os povos indígenas mais investigados estão os Xavante, com cinco produções bibliográficas; os Suruí, com quatro produções e os Kaiangang e Tapirapé, com três publicações cada um.

Entre as técnicas para diagnosticar enteroparasitose, há prevalência da técnica de Lutz (67\%). Entre as demais técnicas observadas nos estudos estão: Faust (21\%), Exame direto (18\%), Willis (18\%), Ritchie (16\%), KatoKatz (14\%), TF-Teste ${ }^{\circledR}$ (8\%), Centrifugo-flutuação (8\%), Baermann\&Moraes (6\%), Harada-Mori (4\%), Coloração de Kinyoun a frio (4\%), Stool \& Hausseer (2\%), Simões Barbosa (2\%), Coprotest ${ }^{\circledR}(2 \%)$, Flutuação com sulfato de zinco (2\%) e Microscopia direta (2\%). Do total de trabalhos encontrados (57 produções), 28 utilizaram mais de uma técnica para o diagnóstico.

Nos trabalhos pesquisados e analisados, dentre os enteroparasitas patogênicos presentes na quase totalidade dos estudos destacam-se os helmintos A. lumbricoides, Ancilostomídeos, T. trichiura, S. stercoralis, Enterobius vermicularis, Hymenolepis nana, Schistosoma mansoni; Taenia saginata e Taenia $s p$ e os protozoários G. lamblia e E. histolytica, Entamoeba coli, Endolimax nana, Balantidium coli, Entamoeba hartmanni, Chilomastix mesnili, lodameba bütschlii, Blastocystis hominis, Pentatrichomonas hominis, Entamoeba hartmanni e Enteromonas hominis.

Durante a sistematização do referencial bibliográfico documental sobre a ocorrência de enteroparasitoses em indígenas no Brasil, foram encontrados quatro trabalhos de revisão bibliográfica, os quais trataram de aspectos gerais dentro da saúde indígena, incluindo o enteroparasitismo como foco principal ou como um dos subtópicos da pesquisa. O primeiro, Epidemiologia das infecções diarreicas entre populações indígenas da Amazônia (LINHARES, 1992), abordou a epidemiologia das infecções diarreicas entre populações indígenas na Amazônia; o segundo, Enteroparasitoses em Populações Indígenas no Brasil: Uma revisão sistemática da literatura científica (VIEIRA, 2003), fez uma revisão sistemática das produções científicas que abordam a 
epidemiologia das enteroparasitoses em populações indígenas no Brasil; o terceiro, Infecções intestinais em populações Indígenas de Rondônia (HAVERROT, ESCOBAR e COIMBRA, 2003), fez um relatório sobre as infecções intestinais no Estado de Rondônia, baseado em dados secundários cedidos pelo Distrito Sanitário Especial Indígena (DSEI) e o quarto, Demandas de Saúde da População Indígena: Uma revisão integrativa (SCHERETTE, 2011), objetivou identificar na literatura brasileira quais são as demandas de saúde da população indígena em um período determinado.

\section{Discussão}

Diversas publicações apresentam informações sobre a epidemiologia de povos indígenas e sobre as peculiaridades das patologias que ocorrem entre essas populações. $O$ perfil de morbimortalidade é um importante parâmetro de avaliação das condições de vida de uma população, sendo resultado da interação de diversos fatores interdependentes (VIEIRA, 2003). O mesmo autor aponta que não é possível separar o nível de morbimortalidade de fatores históricos, socioeconômicos, demográficos, ambientais, culturais e ecológicos, advindos da interação com a sociedade não ameríndia.

As doenças infecciosas e parasitárias persistem como as principais causas de morbimortalidade de indígenas no Brasil (BASTA et al, 2012). Vários estudos sustentam que essas condições são associadas a inadequações e à baixa efetividade dos programas de controle do parasitismo intestinal e das diarreias em área indígena (PALHANO-SILVA, et al, 2009).

Desde 1952 podem ser encontradas produções científicas sobre a temática discutida na presente proposta de pesquisa bibliográfica. Em trabalho semelhante realizado por Vieira (2003), os resultados apresentaram que 0 aumento das produções científicas sobre enteroparasitismo em comunidades indígenas no Brasil ocorre desde a década de 1980.

De acordo com o Censo Demográfico de 2010, mais de $40 \%$ da população indígena do Brasil está na região da Amazônia, enquanto os 
demais grupos étnicos estão divididos pelas demais regiões do país. De acordo com Vieira (2003), a região Norte, seguida da região CentroOeste, tem o maior número de comunidades indígenas e, por isso, apresenta grande número de publicações sobre essas populações. Além disso, o autor afirma que o maior número de grupos étnicos proporciona uma maior preocupação dos pesquisadores quanto às investigações científicas, dentre as quais estão as pesquisas sobre enteroparasitoses.

A região Nordeste, apesar de ser a segunda região com o maior número de indígenas declarados (208.691 indígenas) (IBGE, 2010), foi a que menos apresentou estudos sobre a referida temática, com um total de apenas dois trabalhos. No estado da Bahia, há 21 etnias, das quais 16 são reconhecidas pela Funai (FUNDAÇÃO NACIONAL DO ÍNDIO). A pesquisa de Oliveira et al (2013) Análise comparativa do parasitismo intestinal de ameríndios da etnia Pankararé em três aldeias no Estado da Bahia, Brasil apresentou os resultados sobre a análise de um percentual dos indígenas Pankararé pertencentes às três aldeias nas quais a etnia se divide. Como resultados da pesquisa, foram encontrados $37,1 \%$ de amostras positivas para a presença de enteroparasitas nas 134 amostras analisadas.

$\mathrm{Na}$ extensão nacional, existem cerca de 305 etnias (IBGE, 2010), das quais as mais populosas são: Tikúna (46 mil), Guarani Kaiowá (43,4 mil), Kaingang (37,4 mil), Makuxí (28,9 mil), Terena $(28,8 \mathrm{mil})$ e Tenetehara (24,4 mil). Contrariando os resultados apontados, a etnia que teve maior número de pesquisas desenvolvidas foi a Xavante, seguida por Suruí, Kaiangang e Tapirapé. A facilidade de acesso a essas comunidades pode ter sido um dos pontos positivos para o desenvolvimento dos trabalhos, pois muitos grupos indígenas ainda vivem isolados em áreas de difícil acesso.

As técnicas para o diagnóstico de enteroparasitoses utilizadas nos estudos acessados demonstram grandes diferenças entre si. Estudos comparativos acerca das técnicas coprológicas ressaltam as diferenças de especificidade e de sensibilidade, bem como de outros fatores, como a facilidade de execução e de custo e a capacidade de indicar, da maneira mais expressiva possível, a intensidade do processo infeccioso, em termos individuais e populacionais (Castilho et al, 1984). A técnica de Lutz destaca-se como a mais utilizada entre os trabalhos pesquisados, 
seguida por Faust, Willis, Exame direto, Ritchie e Kato-Katz. As demais técnicas utilizadas foram citadas em um número menor de trabalhos. Resultado semelhante foi encontrado por Vieira (2003), o qual destaca o método de Lutz pela fácil execução e pelo baixo custo. No entanto, a técnica de Lutz apresenta pouca sensibilidade e especificidade para certas espécies parasitárias. Para a determinação s e gu ra de u m diagnóstico parasitológico completo, é necessário o uso de um conjunto de técnicas, o que ocorre em vinte e oito das produções técnicas listadas.

\section{Considerações finais}

O entendimento da epidemiologia da infecção intestinal em comunidades pobres isoladas é importante na elaboração de estratégias eficazes para combater essas doenças tropicais negligenciadas (BRIONESCHÁVEZ et al, 2013). As enteroparasitoses são doenças de baixa prioridade para a saúde pública. Em ambientes rurais, ou em comunidades descaracterizadas pela introdução de costumes diferentes de sua cultura, o contato contínuo com o ambiente poluído propicia a infecção por grande diversidade de parasitos.

A meta-análise mostrou que os estudos sobre enteroparasitoses em comunidades indígenas têm aumentando significativamente nos últimos anos; entretanto, a grande maioria foi desenvolvida na região Norte. Isso demonstra que ainda existe uma escassez de pesquisas sobre a temática nas demais regiões do país, tendo em vista que outras regiões, a exemplo do Nordeste, possui um grande quantitativo de etnias e aldeias distribuídas em sua extensão territorial, e poucos trabalhos vêm sendo desenvolvidos na região.

Mesmo havendo um aumento considerável das produções, quando comparado à importância epidemiológica da temática dentro da saúde, esse número ainda é escasso. É necessário haver maiores pesquisas e estudos sobre as enteroparasitoses nas diversas comunidades indígenas em torno do país, podendo-se, assim, delinear um perfil epidemiológico para futuras ações de prevenção e cuidado da saúde humana e ambiental. 


\section{Referências bibliográficas}

AGUIAR, J. I. A. A. et al. Intestinal protozoa and helminths among Terena Indians in the State of Mato Grosso do Sul: high prevalence of Blastocystis hominis. Revista da Sociedade Brasileira de Medicina Tropical, Minas Gerais, v. 40, n. 6, p. 631-634, 2007.

ANDRADE, R. C. R. et al. Prevalência de parasitos intestinais em ameríndios Kayapós do Estado do Pará, Brasil. Revista Saúde Física \& Mental, Rio de Janeiro, v. 3, n. 2, p. 50-58, 2013.

ASSIS, E. M. Prevalência de enteroparasitoses e condições sanitárias na comunidade indígena Maxakali. 2010. 105 f. Dissertação (Mestrado em Ciências Biológicas) Governador Valadares (MG), Universidade Governador Valadares, [2010].

AYTAI, D. Os cantores da floresta III: notas de Antropologia Física dos índios Mamaindê. Revista da Universidade Católica de Campinas, São Paulo, v. 12, n. 29/30, p. 132-135, 1966.

BASTA P. C.; ORELANA J. D. Y.; ARANTES R. Perfil epidemiológico dos povos indígenas no Brasil: notas sobre agravos selecionados. In: GARNELO, A.; PONTES, L. (Org.). Saúde Indígena: uma introdução ao tema. Brasília, MEC-SECADI, 2012. p. 60107.

BÓIA, M. N. et al. Tuberculose e parasitismo intestinal em população indígena na Amazônia brasileira. Revista de Saúde Pública, São Paulo, v. 43, n. 1, p. 176-8, 2009.

BORGES, J. D. et al. Parasitoses intestinais de indígenas da comunidade Mapuera (Oriximiná, Estado do Pará, Brasil): elevada prevalência de Blastocystis hominis e encontro de Cryptosporidium sp e Cyclospora cayetanensis. Revista da Sociedade Brasileira de Medicina Tropical, Minas Gerais, v. 40, n. 3, p. 348-350, 2009.

BRANDELLI, C. L. C.; DE CARLI, G. A.; MACEDO, A. J. Intestinal parasitism and socio-environmental factors among Mbyá-Guarani indians in Porto Alegre, Rio Grande do Sul, Brazil. Revista do Instituto de Medicina Tropical de São Paulo, São Paulo, v. 54, n. 3, p. 119-122, 2012.

BRIONES-CHÁVEZ, C. et al. Differences in prevalence of geohelminth infections between indigenous and settler populations in a remote Amazonian region of Peru. Tropical Medicine and International Health, Europa, v. 18, n. 5, p. 615-618, 2013.

BRUNO, A. A. G. Condições Sanitárias em Escolares em Zonas Rurais do Território Federal do Amapá. 1978. Dissertação (Mestrado) - Campinas, Universidade de Campinas, [1978]. 
CARDOSO, A. M.; COIMBRA JR., C. E. A.; WERNECK, G. L. Risk factors for hospital admission due to acute lower respiratory tract infection in Guarani indigenous children in southern Brazil: a population-based case-control study. Trop Med Int Health, n. 18, p. 596-607, 2013.

CARME, B. et al. Intestinal parasites among Wayampi Indians from French Guiana. Parasite Journal, v. 9, p. 167-174, 2002.

CARVALHO-COSTA, F. A. et al. Giardia lamblia and other intestinal parasitic infections and their relationships with nutritional status in children in Brazilian Amazon. Revista do Instituto de Medicina Tropical de São Paulo, São Paulo, v. 49, n. 3, p. 147153, 2007.

CARVALHO, G. L. X. Prevalência de enteroparasitoses em crianças de 0 a 12 anos e condições sanitárias na Terra Indígena Xakriabá, São João das Missões, Minas Gerais, 2007. 2011. 115 f. Dissertação (Mestrado em Ciências Biológicas) - Ouro Preto, Universidade Federal de Ouro Preto, [2011].

CASTILHO V. L. P. et al. Exame parasitológico quantitativo das fezes: estudo comparativo entre os métodos de MacMaster, stool-Hausheer e Kato-Katz. Revista da Sociedade Brasileira de Medicina Tropical, n 17, p. 209-212, 1984.

CERQUEIRA D. S.; SILVA M. A.; BOLPATO, M. B. Influência das condições socioeconômicas e ambientais associadas a parasitoses intestinais em população indígena Xavante. Revista Eletrônica da Univar, Mato Grosso, v. 1, p. 148-153, 2013.

CHERNELA J. M.; THATCHER V. E. Comparison of parasite burdens in two native Amazonian populations. Medical Antropology, v. 10, p. 279-285, 1989.

COIMBRA JR C. E. A.; MELLO D. Enteroparasitas e Capillaria sp. entre o grupo Suruí, Parque Indígena Aripuanã, Rondônia. Memórias do Instituto Oswaldo Cruz, Rio de Janeiro, v. 76, n. 3, p. 299-302, 1981.

COIMBRA JR C. E. A.; SANTOS R. V. Avaliação do estado nutricional num contexto de mudança sócio-econômica: o grupo indíegan Suruí do Estado de Rondônia, Brasil. Cadernos de Saúde Pública, Rio de Janeiro, v. 7, n. 4, p. 538-562, 1991 - a.

Parasitismo intestinal entre o grupo indígena Zoró, Estado do Mato Grosso, Brasil. Cadernos de Saúde Pública, Rio de Janeiro, v. 7, n.1, p. 100-103, 1991 - b.

Saúde, minorias e desigualdade: algumas teias de inter-relações, com ênfase nos povos indígenas. Ciênc. Saúde Coletiva, Rio de Janeiro, v.5, p.125-32, 2000.

Perfil epidemiológico da população indígena no Brasil. Documento de trabalho. Universidade Federal de Rondônia, Porto Velho, n 3, p. 1-37, 2001.

COIMBRA JR., C.E.A.; SANTOS, R. V., ESCOBAR, A. L. Epidemiologia e saúde dos povos indígenas no Brasil. Rio de Janeiro: Editora FIOCRUZ, 2005. 
COIMBRA JR., C. E. A.; BASTA, P. C. The burden of tuberculosis in indigenous peoples in Amazonia, Brazil. Thans R Soc Trop Med Hyg, n. 101: p. 635-6, 2007.

COIMBRA JR., C. E. A. Saúde e povos indígenas no Brasil: Reflexões a partir do I Inquérito Nacional de Saúde e Nutrição Indígena 2014. Cad. Saúde Pública, Rio de Janeiro, v. 30, n. 4, p. 855-859, 2014.

CONFALONIERI, U. E. C.; ARAÚJO, A. J.; FERREIRA, L. F. Enteroparasitos em índios Yanomami. Memórias do Instituto Oswaldo Cruz, Rio de Janeiro, v. 84, n. supl. 4, p. 111-113, 1989.

FAGUNDES U. M. Avaliação do Estado Nutricional das crianças Indias do alto Xingu. 1977. Tese (Doutorado em Medicina) - Universidade Federal de São Paulo - USP, São Paulo, [1977].

FERRARI, J. O. et al. Intestinal parasites among Karitiana indians from Rondônia State, Brazil. Revista do Instituto de Medicina Tropical de São Paulo, São Paulo, v. 34, n. 3, p. 223-225, 1992.

FERREIRA, C. S. et al. Intestinal parasites in Iaualapiti Indians from Xingu Park, Mato Grosso, Brazil. Memórias do Instituto Oswaldo Cruz, Rio de Janeiro, v. 86, n. 4, p. 441-442, 1991.

FONTBONNE, A. et al. Fatores de risco para poliparasitismo intestinal em uma comunidade indígena de Pernambuco, Brasil. Cadernos de Saúde Pública, Rio de Janeiro, v. 17, n. 2, p. 367-373, 2001.

GENARO, O.; FERRARONI, J. J. Estudo sobre a malária e parasitoses intestinais em indígenas da tribo Nadeb-Maku, Estado do Amazonas, Brasil. Revista de Saúde Pública, São Paulo, v. 8, p.162-169, 1984.

GILIO, J.; MIORANZA, S. L.; TAKIZAWA, M. G. M. H. Parasitismo intestinal em índios da reserva indígena Rio das Cobras. Revista Brasileira de Análises Clínicas, Rio de Janeiro, v. 38, n.3, p. 193-195, 2006.

HAVERROTH, M.; ESCOBAR, A. L.; COIMBRA JR., C. E. A. Infecções intestinais em Populações Indígenas de Rondônia (Distrito Sanitário Especial indígena Porto Velho). Relatório, Rondônia, n 8, p. 2-18, 2003.

INSTITUTO BRASILEIRO DE GEOGRAFIA E ESTATÍSTICA. Censo Demográfico. Rio de Janeiro: IBGE, 2010.

JUNIOR, C. S. D. et al. Prevalência de parasitoses intestinais e estado nutricional, segundo sexo e idade, entre a população indígena Caxixó, Minas Gerais, Brasil. Revista Brasileira de Estudos da População, Rio de Janeiro, v. 30, n. 2, p. 595-602, 2013. 
KAMEYAMA I. Parasitoses Intestinais entre os índios do Parque Nacional do Xingu. Alguns aspectos epidemiológicos e ecológicos. 1985. 107 f. Dissertação (Mestrado em Saúde Pública) - Faculdade de Saúde Pública na Universidade de São Paulo, São Paulo, SP [1985].

KNIGHT, R.; PRATA, A. Intestinal parasitism in Amerindians at Coari, Brazil. Transactions of Royal Society of Tropical Medicine and Hygiene, Londres, v. 66, p. 809-810. 1972.

KOBAYASHI, J. et al. Prevalence of intestinal parasitic infection in Five farms in Holambra, S. P., Brazil. Revista do Instituto de Medicina Tropical de São Paulo, São Paulo, v. 37, n. 1, p. 13-18, 1995.

LANELLI, R. V.; SILVA, J. P.; AGOSTINI, S. M. Parasitoses intestinais nos índios Xavante de Parabubure, Mato Grosso, Brasil. Cadernos de Saúde Pública, Rio de Janeiro, v. 11, n. 4, p. 629-630, 1995.

LAWRENCE, D. N. et al. Epidemiologic studies among Amerindian populations of Amazonia. The American Journal of Tropical Medicine and Hygiene, Chicago, v. 29, n. 4, p. 530-537, 1980.

LEITE, M. S. et al. Alimentação e nutrição dos povos indígenas no Brasil. In: KAC, G.; SICHIERI, R.; GIGANTE, D. P. (Org.). Epidemiologia nutricional. Rio de Janeiro: Editora Fiocruz, 2007. p. 128-30.

LINHARES, A. C. Epidemiologia das infecções diarreicas entre populações indígenas da Amazônia. Cadernos de Saúde Pública, Rio de Janeiro, v. 8, n. 2, p. 121-128, 1992.

MALHEIROS, A. F. Ocorrência de patógenos intestinais e fatores de risco associados à infecção entre os índios Tapirapé habitantes da Amazônia mato-grossense, Brasil. 2011. 51f. Tese (Doutorado em Biologia da Relação Patógeno-Hospedeiro) - Instituto de Ciências Biomédicas da Universidade de São Paulo, São Paulo, [2011].

MALHEIROS, A. F. et al. Prevalence of Hymenolepis nana in Indigenous Tapirapé Ethnic Group from the Brazilian Amazon. American Journal of Biomedical Research, v. 2, n. 2, p. 16-18, 2014.

MALHEIROS, A. F. et al. Strongyloides stercoralis Infestation in Indigenous Tapirapé Ethnic Group from Mato Grosso State, Brazil. American Journal of Epidemiology and Infectious Disease, v. 2, n. 2, p. 63-65, 2014.

MIRANDA, R. A.; XAVIER F. B.; MENEZES R. C. Parasitismo intestinal em uma aldeia indígena Parakanã, sudeste do Estado do Pará, Brasil. Cadernos de Saúde Pública, Rio de Janeiro, v. 14, n. 3, p. 507-511, 1998.

MIRANDA, R. A. et al. Prevalência de parasitismo intestinal nas aldeias indígenas da Tribo Tembé, Amazônia Oriental Brasileira. Revista da Sociedade Brasileira de Medicina Tropical, Minas Gerais, v. 32, n. 4, p. 389-393, 1999. 
MOHER, D. et al. Preferred reporting items for systematic reviews and meta-analyses: the PRISMA statement. J Clin Epidemiol, v. 62, p. 1006-12, 2009.

MOREIRA, L. E. Prevalência de enteroparasitoses, estado nutricional e condições sanitárias: um estudo na população Krenak, Resplendor, Minas Gerais. 2008. 144f. Dissertação (Mestrado em ciências Biológicas) - Faculdade de Ciências da Saúde, Universidade Vale do Rio Doce, Governador Valadares, [2008].

MOURA, F. T. et al. Enteroparasite contamination in peridomiciliar soils of two indigenous territories, State of Paraná, southern Brazil. Revista Panam Salud Publica, Estados Unidos, v. 27, n. 6, p. 414-22, 2010.

NEEL, J. V. et al. Further studies of the Xavante Indians. VIII. Some observations on blood, urine, and stool specimens. The American Journal of Tropical Medicine and Hygiene, Chicago, v. 17, n. 3, p. 474-485, 1968.

NERES-NORBERG, A. et al. Enteroparasitismo em indígenas Terena em El Estado de Mato Grosso do Sul, Brasil. Revista de Salud Pública, Colômbia, v. 16, n. 6, p. 856$870,2014$.

NEVES, R. E. Estudo das características culturais e sócio-econômicas da aldeia indígena Jaraguá-Ytu, São Paulo, Brasil, correlacionando-as com a prevalência de parasitos intestinais. 2010. 71 f. Dissertação (Mestrado em Ciências) - da Coordenadoria de Controle de Doenças da Secretaria de Estado da Saúde de São Paulo, São Paulo, [2010].

OLIVEIRA, E. P. S. et al. Análise comparativa do parasitismo intestinal de ameríndios da etnia Pankararé em três aldeias no Estado da Bahia, Brasil. Revista InterSciencePlace, Rio de Janeiro, v. 26, n. 1, art. 26, 2013.

OLIVEIRA H. C. O estado de saúde dos índios Karajás em 1950. Revista do Museu Paulista, São Paulo, v. 6 (suplemento), p. 489-508, 1952.

OLIVEIRA, R. A.; GURGEL-GONÇALVES, R.; MACHADO, E. R. Intestinal parasites in two indigenous ethnic groups in northwestern Amazonia. Acta Amazonica, Amazônia, v. 46, n. 3, p. 241-246, 2016.

PALHANO-SILVA, C. S. et al. Intestinal parasitic infection in the Suruí indians. Brazilian Amazon. Interciencia, Venezuela, v. 34, n. 4, 2009.

PARDO M, L. E. et al. Prevalence of intestinal parasitoses in children at the Xingu Indian Reservation. Jornal de Pediatria, Rio de Janeiro, v. 86, n. 6, 2010.

PARDO M, L. E. et al. Prevalence of Helicobacter pylori infection and intestinal parasitosis in children of the Xingu Indian Reservation. Jornal de Pediatria, Rio de Janeiro, v. 87, n. 5, p. 393-398, 2011. 
PEREZ, M. D.; ARTIGAS, P. T.; DEL PONTE, G. Levantamentos parasitológicos, visando em particular a esquistossomose mansônica, realizados em postos indígenas do Estado de São Paulo. Contribuição ao levantamento da carta planorbídica do Estado de São Paulo. Revista de Farmácia e Bioquímica, São Paulo, v.10, n. 2, p. 239-245, 1972.

REY, L. Bases da Parasitologia Médica. Rio de Janeiro: Editora Guanabara Koogan, 2002.

RIOS, L. et al. Prevalência de parasitos intestinais e aspectos socioambientais em comunidade indígena no Distrito de Iauaretê, Município de São Gabriel da Cachoeira (AM), Brasil. Revista Saúde e Sociedade, São Paulo, v. 16, p. 76-86, 2007.

SANTOS, R. V.; COIMBRA JR., C. E. A.; OTT, A. M. T. Estudos epidemiológicos entre os grupos indígenas de Rondônia. III. Parasitoses intestinais nas populações dos vales dos rios Guaporé e Mamoré. Cadernos de Saúde Pública, Rio de Janeiro, v. 1, n. 4, p. 467477, 1985.

SANTOS, R. V. et al. Intestinal parasitism in the Xavante Indians, Central Brasil. Revista do Instituto de Medicina Tropical de São Paulo, São Paulo, v. 37, n. 2, p. 145-148, 1995.

SANTOS, R. V.; COIMBRA Jr., C. E. A. Saúde Indígena. In: Comissão Nacional sobre Determinantes Sociais da Saúde (Org.). As causas sociais das iniquidades em saúde no Brasil. Rio de Janeiro: Editora Fiocruz, 2008. p. 128-30.

SCHERETTE B. S. Demandas de saúde da população indígena: uma revisão integrativa. 2011. 18 f. Monografia (Graduação em Enfermagem) - Universidade Regional do Noroeste do Estado do Rio Grande do Sul, Rio Grande do Sul, [2011].

SCOLARI, C. et al. Prevalence and distribution of soil-transmitted helminth (STH) infections in urban and indigenous schoolchildren in Ortigueira, Sate of Paraná, Brazil: implications for control. Tropical Medicine and International Healthe, Europa, v.3, n. 4, p. 302-307, 2000.

SERAFIM M. G. Hábitos Alimentares e Nível de Hemoglobina em Crianças indígenas Guarani menores de 5 anos, dos Estados de São Paulo e Rio de Janeiro. 1997. 85 f. Dissertação (Mestrado em Medicina) - Escola Paulista de Medicina da Universidade Federal de São Paulo, São Paulo, [1997].

SILVA, G. M. V. Parasitos intestinais na comunidade indígena Xavante de Pimentel Barbosa, Mato Grosso, Brasil. 2010. 79 f. Dissertação ( Programa de Pós-graduação em Ciências na área de Saúde Pública) - Escola Nacional de Saúde Pública Sergio Arouca da Fundação Oswaldo Cruz, Rio de Janeiro, [2010].

SOUZA, M. C.; SCATENA, J. H. G.; SANTOS, R. V. O Sistema de Informação da Atenção à Saúde Indígena (SIASI): criação, estrutura e funcionamento. Cad. Saúde Pública, Rio de Janeiro, v. 23, p. 853-861, 2007. 
TOLEDO, M. J. O. et al. Avaliação de atividades de controle para enteroparasitos em aldeia Kaingáng do Paraná. Revista de Saúde Pública, São Paulo, v. 43, n. 6, p. 981-90, 2009.

VIEIRA, G. O. Enteroparasitoses em Populações Indígenas no Brasil: Uma Revisão Sistemática da Literatura Científica. 2003. 75 f. Dissertação (Mestrado em Ciências na área da Saúde Pública) - Escola Nacional de Saúde Pública da Fundação Oswaldo Cruz, Rio de Janeiro, [2003].

ZARDINELLO, C. D.; RIBEIRO, C. N. M. Estudo comparativo de enteroparasitoses na escola municipal Jorge amado e na Aldeia indígena Avá Guarani. Revista de Biologia e Saúde, Paraná, v. 3, n. 2, p. 61-67, 2009. 